\title{
Criterios \\ El Taller de Restauraciones de la Diputación Foral de Álava
}

Javier Botella Astorqui

Arquitecto. Servicio de Arquitectura de la Diputación Foral de Álava

\section{Resumen}

Se trata de la descripción arquitectónica, mediante texto, planos y fotografias, del Taller de Restauraciones de la Diputación Foral de Álava ubicado en la calle Vitorialanda de Vitoria Gasteiz. El trabajo consistió en aprovechar la estructura de un viejo pabellón industrial para habilitarlo como taller con todos sus espacios necesarios: talleres, laboratorios, salas, biblioteca, aula de formación, despachos, etc. Particular interés tiene el esquema de circulaciones y relaciones del edificio que articulan el conjunto posibilitando una lectura clara y didáctica del mismo y el tratamiento general de espacios y materiales siendo el conjunto más que un mero pabellón industrial, como corresponde al interés cultural de la actividad que aloja.

\section{Palabras clave}

Diputación Foral de Álava

Vitoria

Taller de restauración

Rehabilitación arquitectónica

Proyecto arquitectónico
El desarrollo de cualquier proyecto de Arquitectura comienza siempre por la inmersión en un tema específico: el análisis del objeto del edificio, lo cual puede ser conocido con anterioridad o no por parte del proyectista. En el caso que nos ocupa, la especificidad del encargo fue novedosa para el arquitecto que suscribe, lo que a veces, y sólo a veces, puede constituir una ventaja derivada de la falta de rutinas adquiridas en la resolución de los problemas planteados. Lo anterior sólo puede funcionar bien si te encuentras ante un planteamiento bien hecho, un programa de necesidades bien elaborado y unos interlocutores solventes. Todo esto se dio en este caso, estableciéndose el nivel de relación adecuado con los responsables del Servicio de Restauraciones de la Diputación Foral de Álava. Conviene señalar lo anterior porque muchas veces los órganos responsables de encargar un proyecto arquitectónico no son conscientes de la importancia de elaborar un programa de necesidades bien estudiado, que permita obviar futuros cambios y correcciones sobre la marcha, lo que no sólo redunda en un encarecimiento de la arquitectura producida, sino que afecta muy negativamente a la calidad del resultado.

Teníamos, por tanto, un buen programa, aunque darle forma siempre se puede hacer de mil maneras distintas y se trataba de acertar en la concreción del mismo. También contábamos con un espacio donde construir; en este caso no se trataba de un solar, sino de un gran pabellón recién adquirido en la calle Vitorialanda del Polígono de Ali- Gobeo, en el entorno del Parque Foral de Servicios y cuya superficie excedía a la precisa para el proyecto pudiéndose utilizar la parte necesaria. El hecho de existir el contenedor previamente no constituía un excesivo condicionante dado que su estructura era regular y a base de grandes luces.

Por otro lado, teníamos suficiente presupuesto, lo que no siempre es posible conseguir. Era por tanto difícil que el resultado no fuese bueno a pesar de las dificultades que siempre conlleva la "aventura" de construir.

Lo primero que decidir era cómo plantear la división del pabellón entre el nuevo edificio que se pretendía y la zona de reserva o sobrante del mismo para los fines planteados. Surge la solución a base de eliminar una crujía, lo que permite cumplir varios objetivos: de una parte el pabellón primitivo se transforma en dos, lo que se considera adecuado de cara a un correcto funcionamiento independiente del Servicio de Restauraciones, destinatario del Taller de Restauraciones, respecto a otros usos que en un futuro se ubicasen en el otro pabellón resultante; de otra parte, la calle generada entre los dos pabellones resuelve las exigencias de separación entre pabellones distintos a efectos de protección contra incendios, además de mejorar las posibilidades de aparcamiento y acceso.

De esta manera, el segmento de pabellón inicial a utilizar para Taller de Restauraciones se definió exactamente en función de las necesidades del mismo, teniendo en cuenta que la mayoría de las superficies requeridas debían ubicarse en planta baja, es decir que se podía 


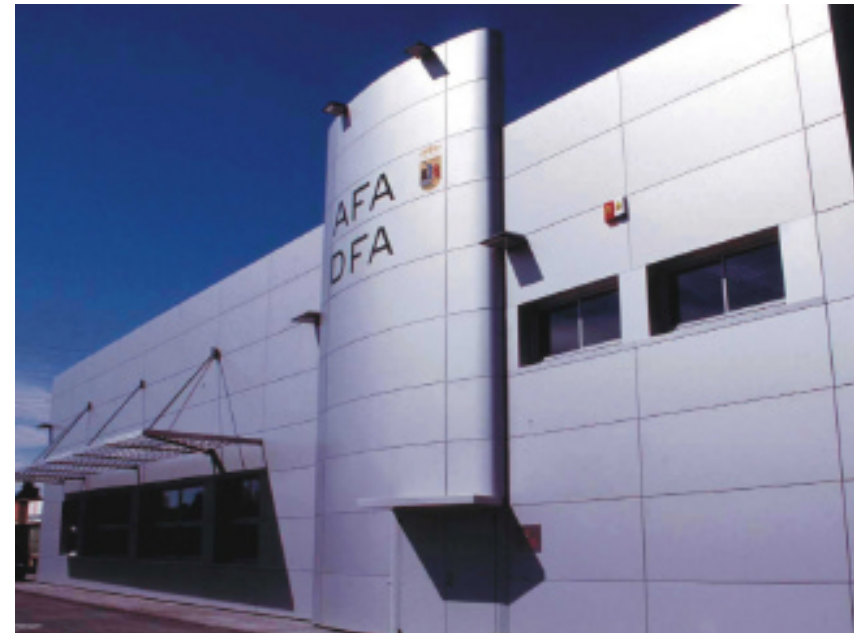

1

realizar una entreplanta, dada la altura existente, para ubicar en la parte superior solamente la parte de despachos.

El programa previsto contemplaba diversos espacios, cada uno de ellos muy específico, englobados en tres grupos: los talleres y almacenes, de mayor superficie y con necesidades de mayor altura, los talleres y laboratorios, con menores requerimientos de superficie y, sobre todo, altura; y la parte de despachos, biblioteca y aula de formación, así como un pequeño archivo de documentos fotográficos. Esta última parte era la que claramente podría ubicarse en la entreplanta por realizar. Como consecuencia de la misma, se generaba un espacio inferior bajo entreplanta de menor altura que el resto del pabellón, adecuado para ubicar los espacios de menor necesidad de altura, tales como el taller de arqueología, los laboratorios de documentos gráficos y fotográficos y de análisis químicos, la zona de tratamiento de maderas y los aseos y vestuarios generales.

Siguiendo este planteamiento, el siguiente punto era buscar el esquema en planta más adecuado que solucionase la distribución de espacios y su interconexión de la forma más racional posible: se resolvió mediante el diseño de un paso longitudinal que separaba a un lado del mismo la zona de talleres laboratorios señalada con menores exigencias de altura y por tanto susceptible de soportar la entreplanta superior mencionada. Al otro lado, los talleres con mayores requerimientos de altura, es decir, los de escultura, pintura, textiles, piedra, almacenes de obras y de materiales y la sala de fotografía y rayos $x$.

Este eje longitudinal enseguida alcanzó un alto nivel de centralidad y protagonismo en el proyecto, no sólo por ser el espacio de acceso principal a los diversos recintos interiores, sino también porque la balconada que se generaba desde la entreplanta contribuía a la visualización e interrelación de los diferentes ámbitos del edificio, posibilitando una lectura clara del mismo y orientando en todo momento a sus usuarios.

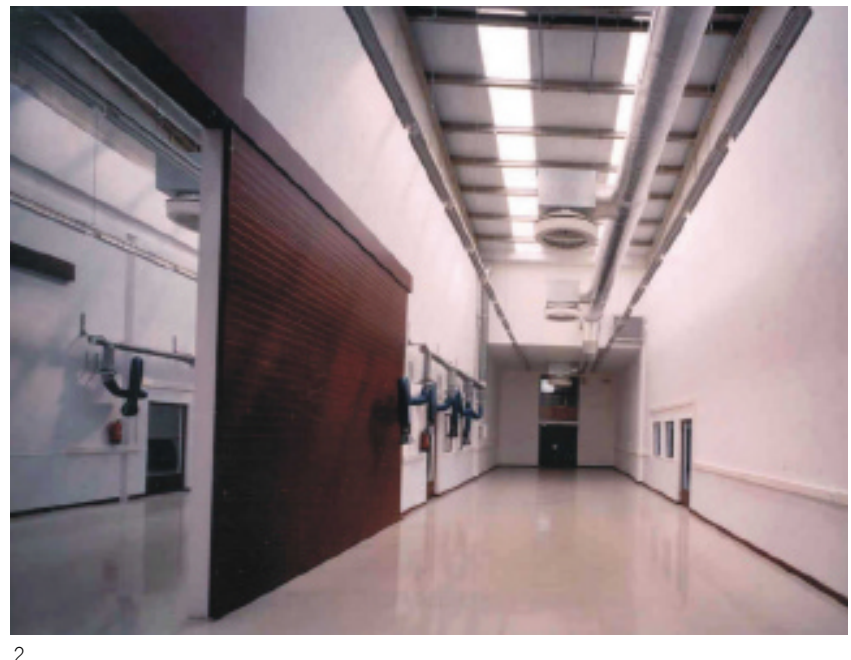

Esta característica de espacio de encuentro y conexión de todas las actividades del edificio, que pronto se perfiló como propio del eje central, quedó potenciado por la apuesta que supone el diseño en planta del mismo con un paramento lineal en la parte de talleres frente a un dibujo en arco de gran amplitud de radio del borde opuesto, es decir, el lado de la balconada; esta disposición, sin apenas incremento en el gasto de superficies respecto a la sección de paso estrictamente necesaria, considero que aporta un sustancial incremento en la capacidad articuladora del eje central y de su condición de lugar relacional del edificio; adicionalmente, el tratamiento de materiales de este espacio a base de un cálido estuco en las paredes, las mamparas acristaladas a juego en tono más oscuro para la distribución de los despachos, la calidad de la barandilla de inox de la balconada y de la escalera de madera de pinotea, el falso techo en celosía que permite el paso de la luz cenital de los luceros de cubierta, y la dignidad de los suelos de resina, comunes a toda la planta baja, así como del linóleo de la entreplanta, contribuyen a definir un espacio personalizado y entiendo que muy adecuado como lugar central del edificio.

De otro lado, las ventanas existentes sobre las puertas de los talleres de más altura, realizadas con vidrio parallamas para separar sectores de incendios, posibilitan la visión de la actividad en el interior de estos talleres desde la entreplanta enfrentada, lo que coadyuva tanto a la interrelación espacial y funcional perseguida como a posibilitar un recorrido didáctico visual sin interferir necesariamente en el trabajo que se está realizando.

Sin embargo, la posición en planta del eje señalado no es ortogonal a ninguna fachada, como cabría esperar de un pabellón con estructura reticular. La razón de su posición semi-diagonal en la planta proviene del hecho de que los espacios requeridos lo eran con diversas cuantías superficiales en función de sus necesidades específicas, lo que llevó a buscar esta diversidad de superficies mediante el giro en la posición del eje central. Automáticamente se generaban espacios, 


\section{Criterios}

El Taller de

Restauraciones de la

Diputación Foral de Álava
3. Planta del pabellón Inicial

4. Planta general del nuevo taller de restauraciones y el pabellón de reservas resultante

\section{Javier Botella Astorqui}

éstos sí siguiendo la trama ortogonal, de diversas cuantías superficiales, lo que posibilitaba dar respuesta a los también diversos metrajes requeridos programáticamente.

Como el segmento de pabellón a utilizar se definía según necesidades, el reparto estaba casi resuelto, sobre todo por encajar las necesidades superficiales de los espacios bajos y sobre la entreplanta ya mencionados. Sin embargo, y en lo que se refería a los talleres de mayor tamaño, su disposición quedaba condicionada por la existencia de la crujía central del edificio con separación entre pilares de seis metros. Era éste un condicionante ineludible que conllevaba el desarrollo de los talleres de forma longitudinal con el ancho mencionado, lo que a la postre se reveló adecuado. Fue muy útil a este respecto, para corroborar la bondad de una distribución por otra parte casi inevitable por la disposición estructural mencionada, el conocimiento de la disposición similar de los talleres del Centro de Intervención del Instituto Andaluz de Patrimonio Histórico de Sevilla cuya experiencia nos sirvió en éste y otros aspectos.

El esquema en planta esbozado se completaba, por una parte, dotando de doble acceso al eje longitudinal desde dos fachadas opuestas, una para acceso de visitas y personal externo, y la otra, más reservada, al personal de la casa; por otra parte, quedaba el muy importante asunto del acceso rodado de vehículos de transporte de materiales y obras, que se resuelve mediante un paso de vehículos que recorre paralelo a la fachada oeste los principales almacenes y talleres, abriéndose, en estos últimos, sucesivos portones deslizantes en el momento de introducir las obras, de manera que pueden ser transportadas a cada taller, y, una vez en el mismo, cambiando perpendicularmente el recorrido, ser desplazadas interiormente ya fuera del vehículo. En el caso del taller de piedra, y dado los pesos a transportar, se dispuso interiormente al recinto de un polipasto eléctrico para el movimiento de la obra.

Un tema muy importante en el edificio que nos ocupa fue la realización de las instalaciones requeridas; en efecto, además de las habi- tuales de electricidad, informática, iluminación, fontaneria, ascensor de minusválidos para la entreplanta, contra incendios y antiintrusión, incluyendo accesos mediante lectores de tarjetas a los principales espacios, se requería una climatización, particularmente en algunos talleres de gran volumen, que por sus altas exigencias en la consecución de niveles de temperatura y grados de humedad adecuados para el mantenimiento de las obras, conllevaron la realización de un sistema de aire acondicionado muy potente (ligado además a la extracción de polvo y otros elementos provocados por la actividad) lo que generó la necesidad de diseñar una amplia entreplanta de servicios encima de los talleres de forma cruzada a los mismos y reduciendo su altura por tanto, aunque sólo en una pequeña parte, en el lado del acceso interior, lo que no les restaba altura en las partes necesarias. Adicionalmente, los talleres reciben luz natural cenital tamizada, evitando las ventanas que pudieran incidir en irradiaciones no deseadas sobre la obra e influyendo en la desestabilización de la climatización. Únicamente en el caso del taller de escultura, para poder trabajar con luz natural, se dispuso una amplia marquesina de lamas de acero inox en la fachada de forma que, sin quitar luminosidad, se evitaba la irradiación directa.

Se pueden enunciar algunos aspectos técnicos de interés del proyecto, desde un punto de vista más especificamente constructivo. Como ya se ha dicho, el edificio del pabellón preexistente sólo condicionó desde el punto de vista de su estructura básica de pilares, vigas y viguetas de hormigón. Todo lo demás fue sustituido por no ser válido para el nuevo uso: en efecto, la cubierta anterior en mal estado fue cambiada por una nueva a base de panel sandwich Perfrisa con aislamiento; la realización de la entreplanta, a base de una losa de hormigón armado vista por su parte inferior, con su cimentación y pilares independientes del resto de la estructura del pabellón, generó la necesidad de ventanas para los laboratorios y despachos lo que, unido a la apertura de huecos de puertas de vehículos en otras fachadas y el ventanal ya aludido del taller de escultura, conllevó la práctica refacción de las fábricas de bloque originales, lo que se so-

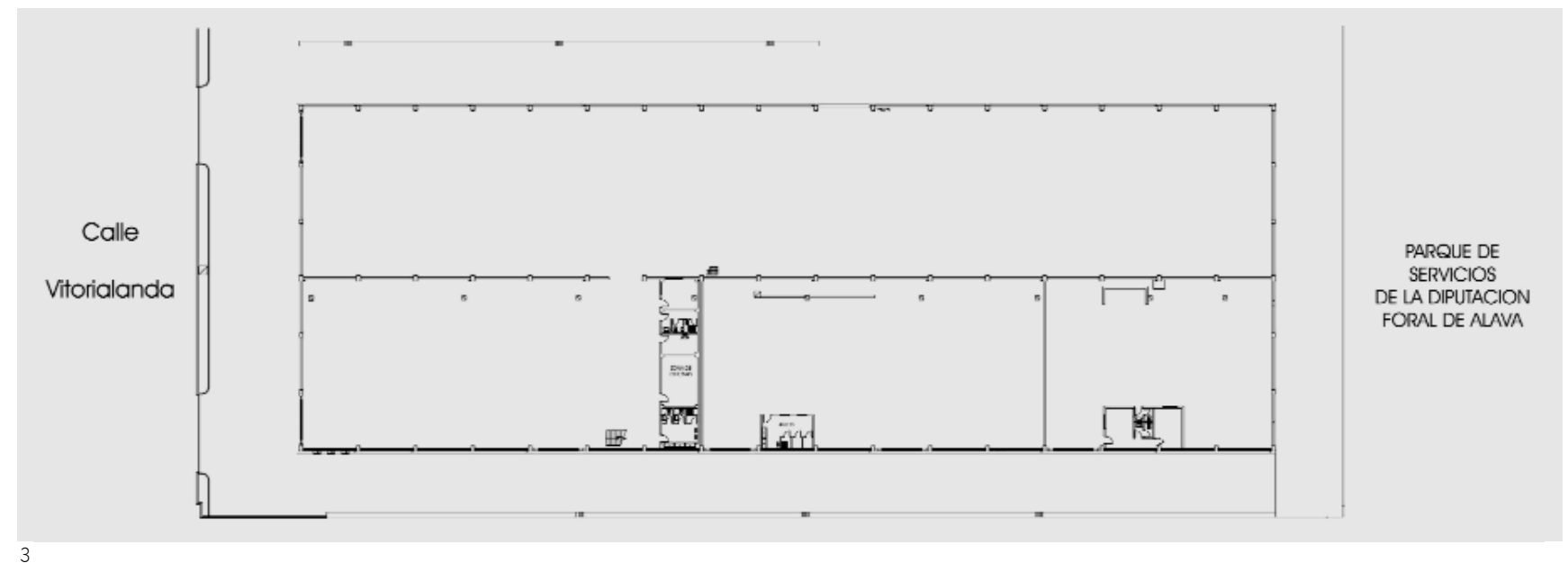

3 
lucionó proyectando un forro de alucobond en la totalidad del edificio creándose una fachada ventilada aislada mediante poliuretano proyectado sobre la fábrica de bloque interior.

Un espacio singular es la Sala destinada a Fotografía y Rayos $X$ donde, para evitar posibles fugas, se proyectaron una losa superior $y$ unos muros perimetrales de hormigón armado además de emplomarse las puertas de acceso.

El resultado de conjunto presenta una gran cohesión formal, con el forro de aluminio mencionado completado con una torreta del mismo material a modo de hito señalizador que contiene los rótulos exteriores sobre la puerta de acceso principal, al lado de la singular marquesina de lamas de inox sobre los ventanales sur, posicionando el conjunto del lado de la calle Vitorialanda, si bien de momento, por razones de seguridad, el acceso se realiza por un enlace rodado trasero que conexiona con el conjunto del Parque de Servicios de Diputación.

El interior, compacto en su articulación y claro de lectura como ya se ha dicho, reúne junto a su condición netamente funcional, obvio primer requisito, un plus de diseño y resolución espacial que hace que el conjunto sea algo más que un mero pabellón, como corresponde a una actividad con el contenido cultural que supone la restauración de elementos del patrimonio artístico.

Ficha técnica

Promotor: Diputación Foral de Álava, como parte del programa de su Dpto. de Cultura

Autor del proyecto y director de las obras: Javier Botella Astorqui, arquitecto del Servicio de Arquitectura de la Diputación Foral de Álava Director de la ejecución material de las obras: Félix Fernández de Larrea, aparejador del Servicio de Arquitectura de la Diputación Foral de Álava

Empresa constructora: Lagunketa S.A.

Fecha de la obra: Enero 2001-Enero 2002
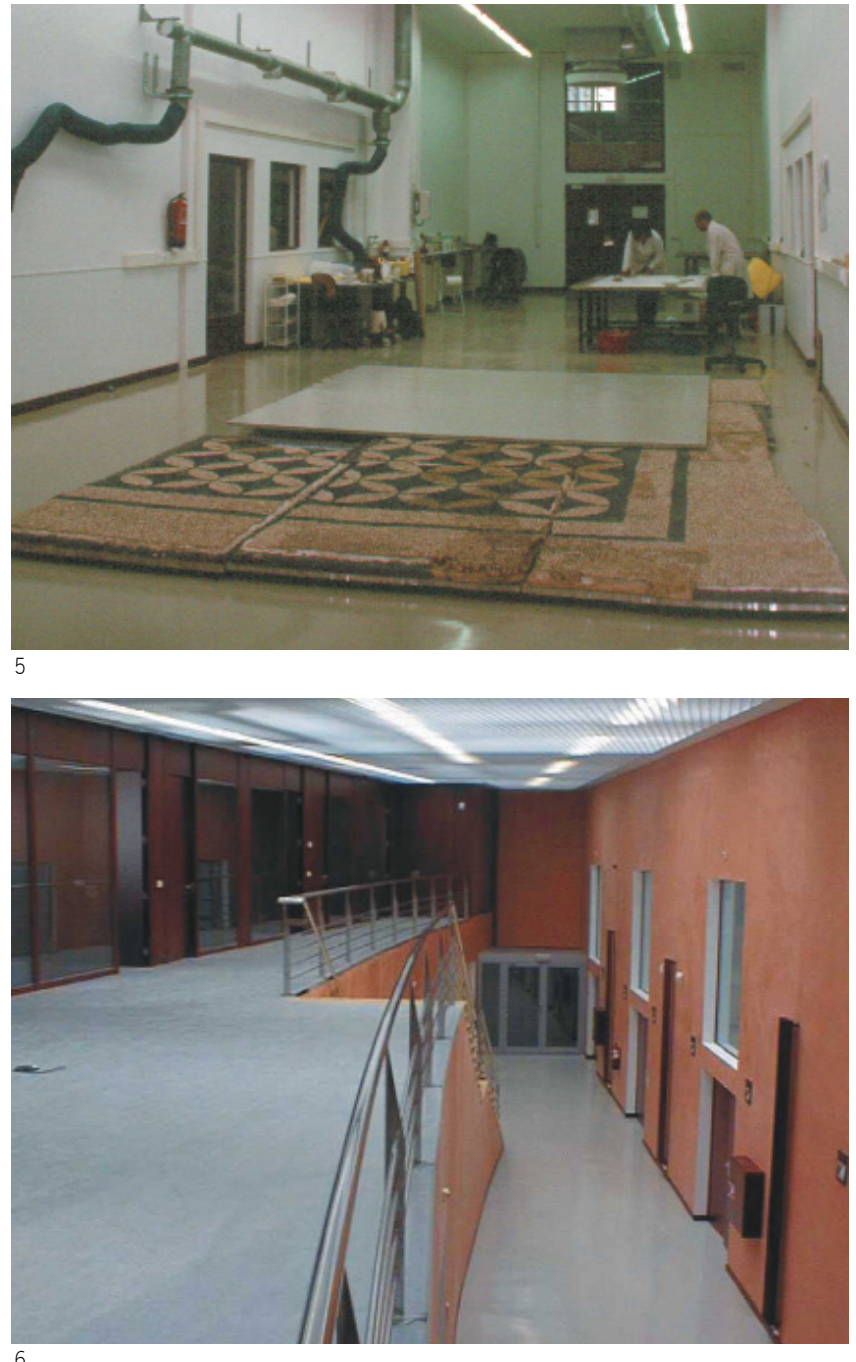

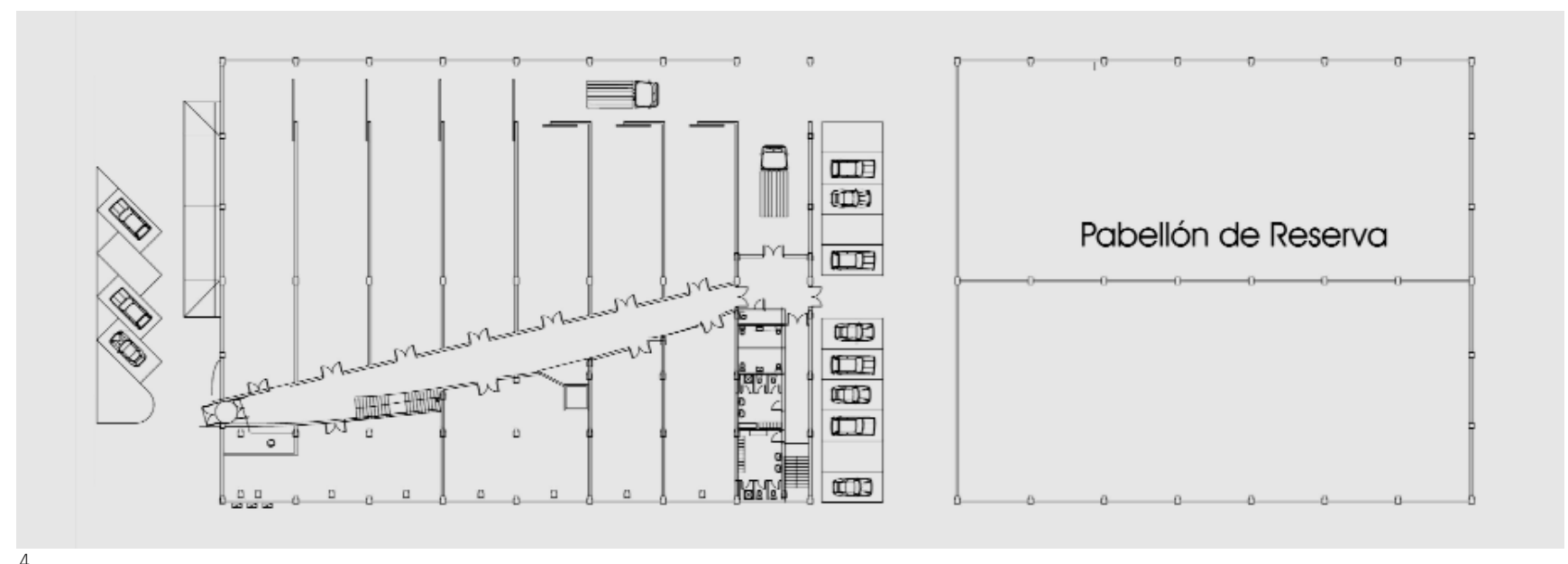

